\title{
Risk factor analysis and management strategies of operating room-related infections after coronary artery bypass grafting
}

\author{
Lifen Pan ${ }^{1}$, Shufang Tan ${ }^{1}$, Linlan $\mathrm{Cao}^{2}$, Xiaoling Feng ${ }^{1}$ \\ ${ }^{1}$ Operating Room, Sun Yat-sen Memorial Hospital, sun Yat-sen University, Guangzhou 510120, China; ${ }^{2}$ Operating Room, First People’s Hospital of \\ Kashi, Kashi 844000, China \\ Contributions: (I) Conception and design: L Pan; (II) Administrative support: S Tan; (III) Provision of study materials or patients: L Cao; (IV) \\ Collection and assembly of data: L Pan, L Cao; (V) Data analysis and interpretation: S Tan, X Feng; (VI) Manuscript writing: All authors; (VII) Final \\ approval of manuscript: All authors. \\ Correspondence to: Shufang Tan. Operating Room, Sun Yat-sen Memorial Hospital, Sun Yat-sen University, Guangzhou 510120, China.
}

Email: tsf_1964@163.com.

Background: To investigate and analyze the risk factors of operating room-related infections after coronary artery bypass grafting (CABG), to propose corresponding infection control management strategies, and to verify and evaluate the effectiveness of the strategy implementation.

Methods: Patients with coronary heart disease who underwent CABG in a hospital from January 2015 to December 2016 were selected for inclusion in this study. The following patient variables were documented: demographics, history of underlying diseases (hypertension and diabetes), preoperative American Society of Anesthesiologists (ASA) score, skin preparation method, perioperative use of antibiotics, operation duration, intraoperative hypothermia, intraoperative blood loss, intraoperative blood transfusion, presence of visitors in the operating room, consecutive use of the same operating room, qualification of the surgeon, and surgical site infection. The infection diagnosis was confirmed using the Diagnostic Criteria for Nosocomial Infections (Trial) issued by the Ministry of Health in 2001. Univariate chi-square tests and multivariate logistic regression analysis were used to analyze the risk factors for infection in patients undergoing CABG, and infection control management strategies were proposed. The proposed infection control strategies were applied to patients who underwent CABG in the hospital in 2017. The effectiveness of the strategy implementation was evaluated.

Results: We analyzed in 139 patients (417 coronary artery bypass grafts from January 2015 to December 2016) and identified 4 surgical site infections. The incidence of infection was $2.88 \%$. According to the univariate analysis, the following factors were related to the high incidence of surgical site infections: advanced age, history of underlying diseases (hypertension and diabetes), obesity, ASA score (class II or above), conventional skin preparation method, irrational perioperative antibiotic use, operation duration $>4$ hours, presence of visitors in the operating room, and consecutive use of the same operating room $(\mathrm{P}<0.05$ for all variables). Multivariate logistic regression analysis showed that advanced age, history of underlying diseases (hypertension and diabetes), irrational perioperative antibiotic use, operation duration $>4$ hours, and presence of visitors in the operating room were high risk factors for surgical site infection after CABG. From January 2017 to December 2017, after applying the proposed infection control measures to patients undergoing CABG, the surgical site infection rate was $1.13 \%$ (3/266), which was significantly different from that of the previous period $(\mathrm{P}<0.05)$.

Conclusions: The following are high risk factors for surgical site infection after CABG: advanced age, history of underlying diseases (hypertension and diabetes), irrational antibiotic use during the perioperative period, operation duration $>4$ hours, and presence of visitors in the operating room. Medical providers should fully understand these risk factors and optimize the adjustable factors during the perioperative period to minimize the incidence of infections after CABG.

Keywords: Coronary heart disease; coronary artery bypass grafting (CABG); surgical site infection; risk factor; 
infection control

Submitted Apr 28, 2018. Accepted for publication Aug 01, 2018.

doi: $10.21037 /$ jtd.2018.08.01

View this article at: http://dx.doi.org/10.21037/jtd.2018.08.01

\section{Introduction}

With the aging of the population, coronary heart disease has become a major cardiovascular disease that affects people's health and is a leading cause of sudden death in patients (1). Coronary artery bypass grafting $(\mathrm{CABG})$ is currently the most effective surgical treatment for coronary heart disease (2). Because patients with coronary heart disease often have severe underlying diseases and CABG surgery involves important organs (such as the heart and great vessels), extensive trauma, and a prolonged operative time, the risk of postoperative nosocomial infection is significantly higher than that in other cardiac operations (3). Surgical site infections after CABG can lead to delayed wound healing, prolonged length of hospital stay, mediastinitis, thorax instability, and involvement of the heart and great vessels, and they can even threaten the lives of patients (4). There are many risk factors responsible for surgical site infections, and these factors can be divided into endogenous and exogenous factors depending on the source. Exogenous factors are more commonly related to operating rooms. Contamination in an operating room is an important cause of nosocomial infections and is the third leading cause of nosocomial infections (5). Effective interventions can help prevent or reduce the incidence of surgical site infections and improve the clinical prognosis. This study aimed to conduct a targeted review of infections in patients undergoing CABG surgery in a hospital and analyze the risk factors for operating room-related infections after CABG. According to these identified risk factors, we developed intervention strategies and investigated the practical significance of using these intervention strategies to reduce surgical site infections in CABG patients in order to provide a reference for clinical practice. We report this work as follows.

\section{Methods}

\section{Clinical data}

From January 2015 to December 2016, 139 patients with coronary artery disease who underwent CABG surgery in a hospital were selected for this study. Of them, 112 were male, and 27 were female, with an average age of $63.86 \pm 18.20$ years (range, $29-82$ years). Surgical site infections were identified in 4 patients, and the incidence of infection was $2.88 \%$. The mean onset time of the infection was $12.20 \pm 10.10$ days (range, $2-30$ days) after surgery. According to the definition of early infection (occurs within 30 days after surgery) by the Centers for Disease Control and Prevention (CDC) in the United States (6), all 139 patients had early infections. We analyzed the cause of infection for each patient and then made corresponding intervention strategies. According to the intervention strategies, the targeted infection control strategies were then applied to 266 patients who underwent CABG in a hospital from January to December 2017.

\section{Infection review and diagnostic criteria}

According to the Standard for Nosocomial Infection Surveillance (7), the following variables were collected and recorded in the CABG Surgical Site Infection Surveillance Registration Form: demographics, history of underlying diseases (hypertension and diabetes), preoperative American Society of Anesthesiologists (ASA) score, skin preparation method, perioperative use of antibiotics, operation duration, intraoperative hypothermia, intraoperative blood loss, intraoperative blood transfusion, presence of visitors in the operating room, consecutive use of the same operating room, qualification of the surgeon, and surgical site infections. The diagnosis of surgical site infections strictly complied with the 2001 Diagnostic Criteria for Nosocomial Infections (Trial) (8), and the infections were divided into 3 categories: superficial incision infections, deep incision infections, and organ/cavity infections. The rationality of the perioperative use of antibiotics was assessed by the Guidelines for clinical use of antibiotics (9).

\section{Infection control management strategies}

(I) Actively treat patients' underlying diseases, control their blood pressure and blood sugar level, improve their nutritional status, and encourage them to quit smoking 
and drinking; (II) perform surgical skin preparation following the 2014 Guideline for the Prevention of Surgical Site Infection before surgery; (III) schedule all patients undergoing surgery in Level 5 (ultra-clean) operating rooms and leave a sufficient interval for room air to be self-cleaned between surgeries scheduled in the same operating room; (IV) have the operating room nurses thoroughly clean the surgical site and the surrounding area before the operation until the disinfection area is large enough. The operating room is divided into an operating area, an anesthesia area, and an instrument station area. Each area is managed by assigned personnel who are not allowed to enter other areas; (V) keep patients warm during the perioperative period; (VI) have the surgical personnel strictly perform the operation using the aseptic technique, and have the circulating nurse watch for breaches in surgical asepsis and coordinate the needs of the surgical team before, during, and after surgery. An infection control professional oversees the regular nosocomial infection control investigations and provides training about the knowledge of nosocomial infections; (VII) control the operating room traffic, including the presence of visitors in the operating room and the frequency of entering and leaving the operating room, and avoid unnecessary moves; (VIII) strengthen surgeons' and nurses' professional skills, improve the coordination of the entire surgical team, and reduce the operation duration; (IX) select the appropriate antibiotics according to the guidelines for clinical use of antibiotics and the list of commonly used antibiotics for surgical prevention and ensure the correct timing and route of administration.

\section{Statistical analysis}

The incidence of surgical site infections before and after infection control management was compared. All data were analyzed using SPSS software version 17.0 (SPSS, Chicago, Illinois, USA). A univariate chi-square test was used to compare the relevant factors. Fisher's exact test was used when the chi-square test was inappropriate. $\mathrm{P}<0.05$ was considered statistically significant. Variables identified as statistically significant by the univariate analysis were included in logistic regression for a multivariate analysis.

\section{Results}

\section{Univariate analysis of surgical site infections after CABG}

The surveillance results for patients undergoing CABG from
January 2015 to December 2016 showed that patient age, history of underlying diseases (hypertension and diabetes), obesity, ASA score, skin preparation method, antibiotic use during the perioperative period, operation duration, presence of visitors in the operating room, and consecutive use of the same operating room were associated with surgical site infections after $\mathrm{CABG}(\mathrm{P}<0.05$ for all variables), while sex, intraoperative hypothermia, intraoperative blood loss, intraoperative blood transfusion, and surgeon qualification were not associated with surgical site infections after CABG ( $\mathrm{P}>0.05$ for each variable, Table 1$)$.

\section{Logistic regression analysis of risk factors for surgical site infection after $C A B G$}

Surgical site infection after CABG was defined as a dependent variable, while age, history of underlying diseases (hypertension and diabetes), obesity, ASA score, skin preparation method, antibiotic use during the perioperative period, operation duration, presence of visitors in the operating room, and consecutive use of the same operating room were defined as independent variables. Logistic regression analysis was performed and showed that advanced age, underlying diseases (hypertension and diabetes), irrational use of antibiotics during the perioperative period, operation duration $>4$ hours, and presence of visitors in the operating room were high risk factors for surgical site infection after CABG (Table 2).

\section{Evaluation of the effectiveness of the infection management strategies}

Interventions for targeted infection control were applied to 266 patients undergoing CABG according to the proposed management strategies in a hospital from January to December 2017. Postoperative surgical site infections were identified in 3 patients. The infection rate was $1.13 \%(3 / 266)$, which was significantly lower than the postoperative infection rate of $2.88 \%$ (4/139) obtained from the patients treated between January 2015 and December $2016(\mathrm{P}<0.05)$.

\section{Discussion}

Surgical site infection is the most common nosocomial infection in patients undergoing surgery and is the focus of nosocomial infection review (10). Surgical site infection after CABG is the consequence of multiple risk factors, 
Table 1 Univariate analysis of surgical site infections after CABG

\begin{tabular}{|c|c|c|c|c|c|}
\hline Variable & $\begin{array}{l}\text { Number of surgical } \\
\text { cases }\end{array}$ & $\begin{array}{l}\text { Number of cases with } \\
\text { infections }\end{array}$ & Incidence (\%) & $\chi^{2}$ & $\mathrm{P}$ \\
\hline Sex & & & & 1.03 & $>0.05$ \\
\hline Female & 27 & 1 & 3.70 & & \\
\hline$\geq 65$ & 59 & 3 & 5.08 & & \\
\hline BMI & & & & 13.83 & $<0.01$ \\
\hline Normal (18.5-23.9) & 69 & 1 & 1.45 & & \\
\hline No & 2 & 0 & 0 & & \\
\hline Yes & 137 & 4 & 2.92 & & \\
\hline ASA score & & & & 8.99 & $<0.01$ \\
\hline Class I & 0 & 0 & 0 & & \\
\hline Class II & 2 & 0 & 0 & & \\
\hline Class III & 54 & 1 & 1.85 & & \\
\hline Class IV & 48 & 1 & 2.08 & & \\
\hline Irrational & 2 & 1 & 50.00 & & \\
\hline Rational & 138 & 3 & 2.17 & & \\
\hline Operation duration (h) & & & & 7.78 & $<0.05$ \\
\hline$\leq 4$ & 56 & 0 & 0 & & \\
\hline$>4$ & 83 & 4 & 4.82 & & \\
\hline Intraoperative hypothermia & & & & 0.58 & $>0.05$ \\
\hline Yes & 99 & 3 & 3.03 & & \\
\hline No & 40 & 1 & 2.50 & & \\
\hline Intraoperative blood loss (mL) & & & & 0.47 & $>0.05$ \\
\hline$\leq 500$ & 106 & 3 & 2.83 & & \\
\hline$>500$ & 33 & 1 & 3.03 & & \\
\hline
\end{tabular}

Table 1 (continued) 
Table 1 (continued)

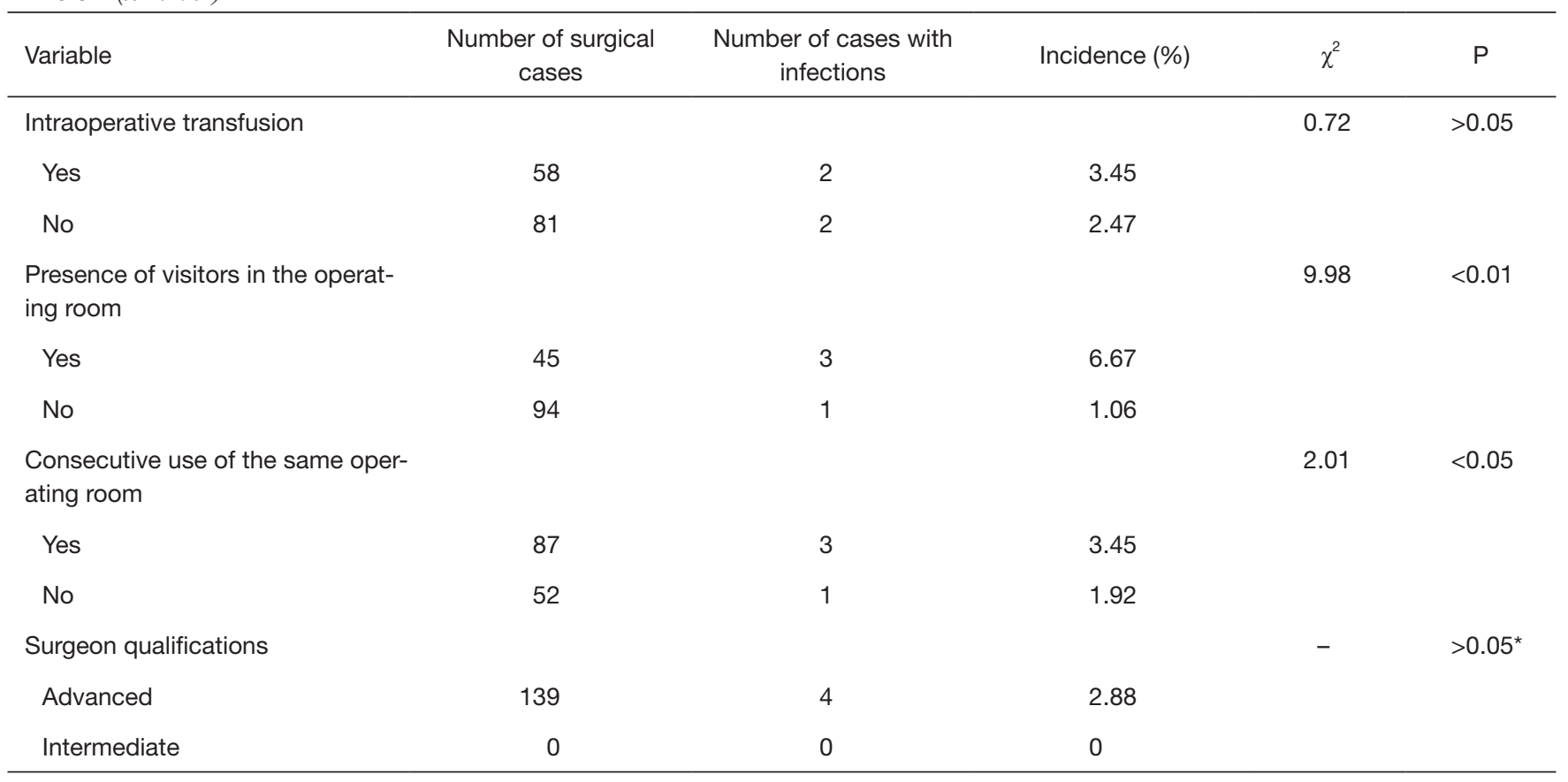

*, Fisher's exact method. CABG, coronary artery bypass grafting.

Table 2 Multivariate logistic regression analysis of risk factors for surgical site infection after CABG

\begin{tabular}{|c|c|c|c|c|c|}
\hline Independent variable & $\beta$ & Wald & $\mathrm{P}$ & OR & $95 \% \mathrm{Cl}$ \\
\hline History of underlying diseases (hypertension and diabetes) & 1.89 & 8.10 & $<0.05$ & 4.97 & $1.66-20.01$ \\
\hline Irrational use of antibiotics during the perioperative period & 1.42 & 0.67 & $<0.01$ & 3.63 & $1.03-12.89$ \\
\hline Operation duration $>4 \mathrm{~h}$ & 1.87 & 0.73 & $<0.01$ & 4.12 & $1.87-26.31$ \\
\hline
\end{tabular}

CABG, coronary artery bypass grafting.

and its mechanism is complex (11). The operating room is an important factor responsible for this type of infection. Analysis of the risk factors for operating room-related infections can help with the development of reasonable management strategies and targeted interventions to reduce the incidence of infections.

Surgical wounds in CABG are clean incisions (Class I). The infection of a clean incision is usually caused by microbial flora on the skin. Thus, it is of great significance to reduce the bacterial population on the patient's skin for the prevention of surgical site infections (12). The conventional method of skin preparation is to clean the surgical site by soap 1 day before surgery. If necessary, a razor is used to remove the hair. However, skin scratches are very common and may become sites for bacterial culture and thereby increase the probability of infection (13). During the perioperative period, the flora on the patient's skin should be reduced based on the maintenance of the skin integrity (14). Appropriate pretreatment of the skin at the surgical site can significantly reduce the incidence of surgical site infections with clean incisions (15). Skin pretreatment methods include preoperative skin cleaning with a chlorhexidine solution (16), removing hair as close as possible to the start of surgery (17), and using a surgical prep razor instead of a regular razor to remove hair $(18,19)$.

The ASA assigns patients into groups defined as Class 
I, II, III, IV, or V based on their physical condition and surgical risk. A higher class indicates a worse physical condition of a patient and is associated with greater surgical risks $(20,21)$. In this study, the univariate analysis showed that a higher ASA score was associated with a higher incidence of surgical site infection, indicating that preoperative general health status is correlated with postoperative anti-infection capacity. If patients also had diabetes, obesity, or hypertension, then their risk of postoperative surgical site infection increased.

Operation duration is closely related to surgical site infections. CABG requires a longer operative time and longer incisions (usually two surgical incisions: a median sternotomy incision and a long saphenous vein harvesting in the legs). Accordingly, the opportunity and routes of exposure of the wounds to bacteria increase. If there were Staphylococcus aureus, Escherichia coli and Acinetobacter baumannii in the air of the operating room, then the incision would be a good medium for these pathogens to grow. Moreover, body surface flora may also migrate into the surgical wounds (22). In this study, both univariate and multivariate analyses showed that operation duration was a risk factor for surgical site infection after CABG. With a prolonged operative time, long-time exposure of the wounds increases the risk of contamination. In addition, consecutive use of the same operating room and visitors were associated with an increased risk of surgical site infection after CABG. If there were visitors in the operating room or if the same operating room was used consecutively for multiple operations, the increased risk of surgical site infection after CABG was consistent with that in the literature (23).

The rational use of antibiotics during the perioperative period can significantly reduce postoperative surgical site infections (24). A study by Wang et al. (5) has shown that the long-term use of antibiotics after surgery is associated with an increased incidence of surgical site infections and antibiotic-resistant infections. In this study, two patients failed to use antibiotics rationally. One patient had incorrect timing for the preventive use of antibiotics before surgery, while antibiotics were improperly selected in the other patient, and the course of the antibiotics was longer than that required by the guideline. The irrational use greatly increased the incidence of surgical site infections after CABG.

Focusing on the risk factors for surgical site infections after $\mathrm{CABG}$, we developed reasonable management strategies. The following interventions may effectively reduce the incidence of surgical site infections: (I) raise the infection control awareness and recognition of the medical staff in the operating room to improve work responsibility during the operation and to strictly implement the protocol; (II) perform a comprehensive physical examination and assessment of the patient before surgery to maintain the patient's stable physical condition throughout the perioperative period; for example, controlling blood sugar, blood pressure, maintaining proper nutrition, and preventing respiratory diseases; (III) ensure that the surgical instruments are managed appropriately; i.e., all surgical instruments shall be managed in the supply room for cleaning, packaging, and sterilization to reduce operating room-related infections; (IV) surgical personnel shall strictly perform an aseptic operation, and the circulating nurse should watch for breaches in surgical asepsis and coordinate the needs of the surgical team before, during, and after surgery; (V) avoid the consecutive use of the same surgical room as much as possible. If inevitable, then close the operating room, and let the air be selfcleaned for at least 15 minutes between operations; (VI) limit the number of medical staff in the operating room, arrange a surgery webinar if there are too many visitors to control the operating room traffic, and avoid unnecessary moves in the operating room; (VII) reduce the operation duration by improving the professional skills of surgeons and assigning specialized surgical assistant nurses who are familiar with the surgical procedures, can closely follow the progress of the procedure, and actively cooperate with the surgeons; (VIII) the rational use of antibiotics during the perioperative period is necessary. Considering the patient's own risk factors, antibiotic resistance, and operation duration, the timing of antibiotic administration and the course of medication should be standardized. Preoperative antibiotic prophylaxis was performed within 0.5 to 2 hours before surgery or at the beginning of anesthesia. If the operative time is longer than 3 hours or the amount of blood loss is more than $1,500 \mathrm{~mL}$, then a dose of antibiotics can be repeated. The total time of antibiotic prophylaxis should be less than 24 hours and may be as long as 48 hours according to the condition of each individual patient (9). This study has verified that targeted interventions can significantly reduce the incidence of surgical site infections after CABG. However, the limitations of this study include a small sample size, a short study time, and the occurrence of surgical site infections in few patients. That being said, these interventions still need further verification to provide stable and feasible infection control management solutions 
based on practical clinical conditions.

In summary, multiple risk factors are involved in surgical site infections after CABG. Some factors are independent, but most factors are correlated to or affect each other. Medical providers shall fully understand these risk factors and optimize those adjustable factors during the perioperative period to minimize the incidence of surgical site infections after CABG.

\section{Acknowledgements}

Funding: Guangdong special fund for public welfare study and ability construction (2014A020209020).

\section{Footnote}

Conflicts of Interest: The authors have no conflicts of interest to declare.

Ethical Statement: This study was approved by the Medical Ethics Committee of Sun Yat-sen University (approval No.: SYXEC-KY-KS-2018-047).

\section{References}

1. Arsenault BJ, Puri R. Reducing exposure to cardiovascular risk factors: the legacy of prevention. J Thorac Dis 2016;8:2340-3.

2. Bonatti J, Ramahi J, Hasan F, et al. Long-term results after robotically assisted coronary bypass surgery. Ann Cardiothorac Surg 2016;5:556-62.

3. Atchley KD, Pappas JM, Kennedy AT, et al. Use of administrative data for surgical site infection surveillance after congenital cardiac surgery results in inaccurate reporting of surgical site infection rates. Ann Thorac Surg 2014;97:651-7; discussion 657-8.

4. King C, Aylin P, Moore LS, et al. Syndromic surveillance of surgical site infections--a case study in coronary artery bypass graft patients. J Infect 2014;68:23-31.

5. Wang F, Cheng J. Antibiotics resistance in the surgery wards of Fuwai Cardiovascular Hospital. Chinese Journal of Nosocomiology 2010;20:116-8.

6. Pull ter Gunne AF, Hosman AJ, Cohen DB, et al. A methodological systematic review on surgical site infections following spinal surgery: part 1: risk factors. Spine (Phila Pa 1976) 2012;37:2017-33.

7. Ministry of Health of the People's Republic of China. WS/ T312-2009 Standard for nosocomial infection surveillance.
Beijing, 2009. Available online: http://www.nhfpc.gov.cn/ cmsresources/mohyzs/cmsrsdocument/doc5842.pdf

8. Ministry of Health of the People's Republic of China. Diagnostic criteria for nosocomial infections (Trial). Beijing, 2001. Available online: http://www.nhfpc.gov.cn/ yzygj/s3593/200804/e19e4448378643a09913ccf2a055c79 d.shtml

9. Ministry of Health of the People's Republic of China. Guidelines for the clinical use of antibiotics. Beijing, 2015. Available online: http://www.nhfpc.gov.cn/ewebeditor/upl oadfile/2015/09/20150928170007470.pdf

10. Hu B, Ge M, Kuan S. Best practice for prevention and control of surgical site infection. Shanghai: Shanghai Scientific and Technical Publishers, 2012:35.

11. Olsen MA, Lock-Buckley P, Hopkins D, et al. The risk factors for deep and superficial chest surgical-site infections after coronary artery bypass graft surgery are different. J Thorac Cardiovasc Surg 2002;124:136-45.

12. Lefebvre A, Saliou P, Lucet JC, et al. Preoperative hair removal and surgical site infections: network metaanalysis of randomized controlled trials. J Hosp Infect 2015;91:100-8.

13. Tu F, Jia Y, Liu L. Effect of different methods of skin preparation on postoperative infections in patients treated in general surgery units. Chinese Journal of Nosocomiology 2015;25: 3771-3.

14. Webster J, Osborne S. Preoperative bathing or showering with skin antiseptics to prevent surgical site infection. Cochrane Database Syst Rev 2012;(9):CD004985. Update in: Cochrane Database Syst Rev 2015;(2):CD004985.

15. Zhang J, Wang L, Ma W, et al. Investigation and control of the epidemic of surgical site infection following clean wound operation in orthopedics patients. Chinese Journal of Infection Control 2014;13: 399-401, 414.

16. Nishihara Y, Kajiura T, Yokota K, et al. Evaluation with a focus on both the antimicrobial efficacy and cumulative skin irritation potential of chlorhexidine gluconate alcoholcontaining preoperative skin preparations. Am J Infect Control 2012;40:973-8.

17. Tanner J, Woodings D, Moncaster K. Preoperative hair removal to reduce surgical site infection. Cochrane Database Syst Rev 2006;(3):CD004122.

18. Mangram AJ, Horan TC, Pearson ML, et al. Guideline for prevention of surgical site infection, 1999. Hospital Infection Control Practices Advisory Committee. Infect Control Hosp Epidemiol 1999;20:250-78; quiz 279-80.

19. Saiman L, Siegel JD, LiPuma JJ, et al. Infection prevention and control guideline for cystic fibrosis: 2013 update. 
Infect Control Hosp Epidemiol 2014;35 Suppl 1:S1-S67.

20. Schoenfeld AJ, Carey PA, Cleveland AW 3rd, et al.

Patient factors, comorbidities, and surgical characteristics that increase mortality and complication risk after spinal arthrodesis: a prognostic study based on 5,887 patients. Spine J 2013;13:1171-9.

21. Meng F, Cao J, Meng X. Risk factors for surgical site infections following spinal surgery. J Clin Neurosci 2015;22:1862-6.

22. Cheng L, Ayi G, Li J, et al. Risk factors for surgical site

Cite this article as: Pan L, Tan S, Cao L, Feng X. Risk factor analysis and management strategies of operating room-related infections after coronary artery bypass grafting. J Thorac Dis 2018;10(8):4949-4956. doi: 10.21037/jtd.2018.08.01 infection in patients undergoing cardiac surgery. Chinese Journal of Infection Control 2017;16: 62-5.

23. Tully L, Gates S, Brocklehurst P, et al. Surgical techniques used during caesarean section operations: results of a national survey of practice in the UK. Eur J Obstet Gynecol Reprod Biol 2002;102:120-6.

24. Meredith DS, Kepler CK, Huang RC, et al. Postoperative infections of the lumbar spine: presentation and management. Int Orthop 2012;36:439-44. 